\title{
ACTION OF FINITE GROUPS ON REES ALGEBRAS AND GORENSTEINNESS IN INVARIANT SUBRINGS
}

\author{
by SHIN-ICHIRO IAI
}

(Received 20th July 1997)

Let $G$ be a finite group of order $N$ and assume that $G$ acts on a Cohen-Macaulay local ring $A$ as automorphisms of rings. Let $N$ be a unit in $A$. For a given $G$-stable ideal $I$ in $A$ we denote by $\mathcal{R}(I)=\oplus_{n \geq 0} I^{n}$ and $\mathcal{G}(I)=\oplus_{n \geq 0} I^{n} / I^{n+1}$ the Rees algebra and the associated graded ring of $I$, respectively. Then $G$ naturally acts on $\mathcal{R}(I)$ and $\mathcal{G}(I)$ too. In this paper the conditions under which the invariant subrings $\mathcal{R}(I)^{C}$ of $\mathcal{R}(I)$ are Cohen-Macaulay and/or Gorenstein rings are described in connection with the corresponding ring-theoretic properties of $\mathcal{G}(I)^{c}$ and the a-invariants $a\left(\mathcal{G}(I)^{c}\right)$ of $\mathcal{G}(I)^{c}$. Consequences and some applications are discussed.

1991 Mathematics subject classification: Primary 13A30; Secondary $13 \mathrm{H} 10$.

\section{Introduction}

Let $A$ be a commutative ring and $G$ a finite group of order $N$. We assume $G$ acts on $A$ as automorphisms of rings. Let $t$ be an indeterminable over $A$. For each ideal $I(I \neq A)$ in $A$ we put

$$
\begin{aligned}
\mathcal{R}(I) & =A[I t] \subseteq A[t] \\
\mathcal{R}^{\prime}(I) & =A\left[I t, t^{-1}\right] \subseteq A\left[t, t^{-1}\right], \text { and } \\
\mathcal{G}(I) & =\mathcal{R}^{\prime}(I) / t^{-1} \mathcal{R}^{\prime}(I)
\end{aligned}
$$

and call them the Rees algebra, the extended Rees algebra, and the associated graded ring of $I$, respectively. Now let us extend the action of $G$ on $A$ to that on the Laurent polynomial ring $B=A\left[t, t^{-1}\right]$, letting $\sigma(t)=t$ for all $\sigma \in G$. Then if the ideal $I$ is $G$ stable that is $\sigma(I) \subseteq I$ for any $\sigma \in G$, the algebras $\mathcal{R}(I)$ and $\mathcal{R}^{\prime}(I)$ remain stable in $B$ under this action of $G$, so that our group $G$ naturally acts on the associated graded ring $\mathcal{G}(I)$ too. In this paper we are interested in the question how and why certain ringtheoretic properties of $\mathcal{R}(I)^{G}$ are determined by those of $\mathcal{G}(I)^{G}$. And our starting point for this research is the following.

Theorem 2.4. Let $A$ be a Cohen-Macaulay local ring and let $I(\neq A)$ be a G-stable ideal in $A$. Assume the order $N$ of $G$ is invertible in $A$. Then if $\mathrm{ht}_{A} I \geq 1$ (resp. $\left.\mathrm{ht}_{A} I \geq 2\right)$, the following two conditions are equivalent. 
(1) $\mathcal{R}(I)^{G}$ is a Cohen-Macaulay (resp. Gorenstein) ring.

(2) $\mathcal{G}(I)^{G}$ is a Cohen-Macaulay (resp. Gorenstein) ring and $a\left(\mathcal{G}(I)^{G}\right)<0$ (resp. $\left.a\left(\mathcal{G}(I)^{G}\right)=-2\right)$.

Here $a\left(\mathcal{G}(I)^{G}\right)$ denotes the a-invariant of $\mathcal{G}(I)^{G}$.

Now let $A$ be a Cohen-Macaulay local ring with maximal ideal $m$ and $d=\operatorname{dim} A \geq 2$. We assume the order $N$ of $G$ is invertible in $A$. Then in general the ring $A$ contains numerous $G$-stable ideals and eventually our question is very subtle to handle. Therefore to go farther, in this paper we would like to restrict our attention mainly to the case where $I=\mathfrak{m}$. Let

$$
\mathcal{R}=\mathcal{R}(\mathfrak{m}) \text { and } \mathcal{G}=\mathcal{G}(\mathfrak{m})
$$

And with this notation we have from Theorem 2.4 the following.

Theorem 3.4. Let $\mathcal{G}$ be a Gorenstein ring and suppose that $G$ trivially acts on the residue class field $k=A / \mathfrak{m}$ of $A$. Consider the following three conditions.

(1) $\mathcal{R}^{G}$ is a Gorenstein ring.

(2) $\mathcal{G}^{G}$ is a Gorenstein ring of $a\left(\mathcal{G}^{G}\right)=-2$.

(3) $\chi_{G, g}=1$ and $a(\mathcal{G})=-2$.

Then one has the implications (1) $\Leftrightarrow(2) \Leftarrow(3)$. Furthermore, if $a(\mathcal{G}) \leq-2$ or if $\mathcal{G}$ is $a$ normal ring and the extension $\mathcal{G} / \mathcal{G}^{G}$ is divisorially unramified, then the above three conditions are equivalent to each other.

We shall briefly recall in Section 3 the definition and some basic properties of the canonical character $\chi_{G, G}$ stated in condition (3) in Theorem 3.4. Instead let us note here two consequences of Theorem 3.4 .

Corollary 3.5. Assume that $\mathcal{R}$ is a Gorenstein ring and that $G$ trivially acts on the residue class field $k=A / \mathrm{m}$ of $A$. Then the following two conditions are equivalent.

(1) $\mathcal{R}^{G}$ is a Gorenstein ring.

(2) $\chi_{G, g}=1$.

Corollary 3.6. Suppose that $A$ is a regular local ring and $G$ trivially acts on the residue class field $k=A / \mathrm{m}$. Let $\rho: G \rightarrow G L\left(\mathrm{~m} / \mathrm{m}^{2}\right)$ be the representation of $G$ over $k$ which is induced from the action on $A$. Then the following two conditions are equivalent.

(1) $\mathcal{R}^{G}$ is a Gorenstein ring.

(2) $\operatorname{dim} A=2$ and $\rho(\mathcal{G}) \subseteq S L\left(\mathrm{~m} / \mathrm{m}^{2}\right)$. 
We will prove Theorem 2.4 in Section 2 . The proof is directly based on the recent progress [4] due to Goto and Nishida in the theory of Rees algebras associated to filtrations of ideals. To check the implications stated in Theorem 3.4 we need a part of the theory of canonical characters $\chi_{G, g}$, that we shall briefly recall in Section 3 . The proof of Theorem 3.4 and its consequences also shall be given in Section 3. In Section 4 we will explore a few examples to illustrate our theorems.

In what follows let $G$ be a finite group of order $N$ which acts on a commutative ring $A$ as automorphisms of rings. We extend the action of $G$ to that on the Laurent polynomial ring $A\left[t, t^{-1}\right]$ with $\sigma(t)=t$ for all $\sigma \in G$.

\section{Proof of Theorem 2.4}

Let $I$ be a $G$-stable ideal in $A$. We put $B=A\left[t, t^{-1}\right], \mathcal{R}=\mathcal{R}(I), \mathcal{R}^{\prime}=\mathcal{R}^{\prime}(I)$, and $\mathcal{G}=\mathcal{G}(I)$. Then $G$ acts on the rings $B, \mathcal{R}, \mathcal{R}^{\prime}$, and $\mathcal{G}$. For each $i \in \mathbb{Z}$ let $F_{i}=I^{i} \cap A^{G}$. Then the family $\mathcal{F}=\left\{F_{i}\right\}_{i \in Z}$ of ideals in $A^{G}$ satisfies the following.

Lemma 2.1. (1) $F_{i}=A^{G}$ for $i \leq 0$.

(2) $F_{i} F_{j} \subseteq F_{i+j}$ for all $i, j \in \mathbb{Z}$.

We put $\mathcal{R}(\mathcal{F})=\sum_{i \geq 0} F_{i} t^{i} \subseteq A^{G}[t]$ and $\mathcal{R}^{\prime}(\mathcal{F})=\sum_{i \in \mathbf{Z}} F_{i} t^{i} \subseteq A^{G}\left[t, t^{-1}\right]$. Then $\mathcal{R}(\mathcal{F})$ and $\mathcal{R}^{\prime}(\mathcal{F})$ are graded $A^{G}$-subalgebras of $A^{G}\left[t, t^{-1}\right]$. Let $\mathcal{G}(\mathcal{F})=\mathcal{R}^{\prime}(\mathcal{F}) / t^{-1} \mathcal{R}^{\prime}(\mathcal{F})$.

Proposition 2.2. (1) $\mathcal{R}^{G}=\mathcal{R}(\mathcal{F})$ and $\mathcal{R}^{\prime G}=\mathcal{R}^{\prime}(\mathcal{F})$ as graded $A^{G}$-algebras.

(2) Suppose that $N$ is invertible in $A$. Then there is a natural isomorphism

$$
\mathcal{G}^{G} \cong \mathcal{G}(\mathcal{F})
$$

of graded $A^{G}$-algebras.

Proof. (1) This follows from the fact that $\mathcal{R}^{G}=\mathcal{R} \cap A^{G}[t]$ and $\mathcal{R}^{\prime G}=\mathcal{R}^{\prime} \cap A^{G}\left[t, t^{-1}\right]$.

(2) Since $N$ is invertible in $A$, from the exact sequence $0 \rightarrow \mathcal{R}^{\prime}(1) \stackrel{t^{-1}}{\rightarrow} \mathcal{R}^{\prime} \stackrel{\bullet}{\rightarrow} \mathcal{G} \rightarrow 0$ of graded $\mathcal{R}^{\prime}$-modules we get the exact sequence

$$
0 \rightarrow \mathcal{R}^{\prime G}(1) \stackrel{t^{-1}}{\rightarrow} \mathcal{R}^{G} \stackrel{g}{\rightarrow} \mathcal{G}^{G} \rightarrow 0
$$

of graded $\mathcal{R}^{\prime G}$-modules, where $\varepsilon$ denotes the canonical epimorphism. (For $x \in \mathcal{R}^{\prime}$ let $x^{*}=x \bmod t^{-1} \mathcal{R}^{\prime}$. Then for each $x^{*} \in \mathcal{G}^{G}$ the element $\left[\sum_{\sigma \in G} \sigma(x)\right] / N$ of $\mathcal{R}^{\prime G}$ is chosen to be the inverse image of $x^{*}$.) Hence $\mathcal{G}^{G} \cong \mathcal{R}^{\prime}(\mathcal{F}) / t^{-1} \mathcal{R}^{\prime}(\mathcal{F})=\mathcal{G}(\mathcal{F})$ by $(1)$.

Lemma 2.3. (1) $A$ in integral over $A^{G}$. Hence $\operatorname{dim} A=\operatorname{dim} A^{G}$ and if $A$ is a local ring, then so is $A^{G}$.

(2) Let $A$ be a Cohen-Macaulay local ring and assume that $N$ is invertible in $A$. Then $\mathrm{ht}_{A} I=\mathrm{ht}_{A} G I^{G}$. 
Proof. (1) For each $a \in A$ let $f_{a}(t)=\prod_{\sigma \in G}(t-\sigma(a))$. Then $f_{a}(t) \in A^{G}[t]$ and $f_{a}(a)=0$. Hence $A$ is integral over $A^{G}$ and so $\operatorname{dim} A=\operatorname{dim} A^{G}$. See [2, (5.8)] for the second assertion.

(2) By [8, Proposition 13] the ring $A^{G}$ is Cohen-Macaulay. Since our ideal $I$ is $G$ stable, the group $G$ acts on the local ring $A / I$ as automorphisms of rings. We have $(A / I)^{G} \cong A^{G} / I^{G}$, because $N$ is invertible in $A$. Hence

$$
\operatorname{dim} A=\operatorname{dim} A^{G} \quad \text { and } \quad \operatorname{dim} A / I=\operatorname{dim}(A / I)^{G}=\operatorname{dim} A^{G} / I^{G}
$$

by (1). On the other hand, since both the local rings $A$ and $A^{G}$ are Cohen-Macaulay, we get

$$
\operatorname{dim} A=\operatorname{dim} A / I+\mathrm{ht}_{A} I \quad \text { and } \quad \operatorname{dim} A^{G}=\operatorname{dim} A^{G} / I^{G}+\mathrm{ht}_{A^{G}} I^{G} .
$$

Thus ht ${ }_{A} I=\mathrm{ht}_{A} I^{G}$.

The next result plays a key role in this paper.

Theorem 2.4. Let $A$ be a Cohen-Macaulay local ring and $I(\neq A)$ a G-stable ideal of A. Assume the order $N$ of $G$ is invertible in $A$. Then if $\mathrm{ht}_{A} I \geq 1$ (resp. $\mathrm{ht}_{A} I \geq 2$ ), the following conditions are equivalent.

(1) $\mathcal{R}^{G}$ is a Cohen-Macaulay (resp. Gorenstein) ring.

(2) $\mathcal{G}^{G}$ is a Cohen-Macaulay (resp. Gorenstein) ring of $a\left(\mathcal{G}^{G}\right)<0\left(\right.$ resp. $\left.a\left(\mathcal{G}^{G}\right)=-2\right)$.

Here $a\left(\mathcal{G}^{G}\right)$ denotes the a-invariant of $\mathcal{G}^{G}$.

Proof. By [8, Proposition 13] and (2.3) $A^{G}$ is a Cohen-Macaulay local ring and $\mathrm{ht}_{A} I=\mathrm{ht}_{A} G I^{G}$. Since $F_{1}=I^{G}$, we get $\mathrm{ht}_{A} G F_{1} \geq 1$ (resp. ht $A_{A} F_{1} \geq 2$ ) if $\mathrm{ht}_{A} I \geq 1$ (resp. $\mathrm{ht}_{A} I \geq 2$ ). Therefore by [4, Part II, (1.2) and (1.4)], provided ht ${ }_{A} I \geq 1$ (resp. ht ${ }_{A} I \geq 2$ ), $\mathcal{R}(\mathcal{F})$ is a Cohen-Macaulay (resp. Gorenstein) ring if and only if $\mathcal{G}(\mathcal{F})$ is a CohenMacaulay (resp. Gorenstein) ring and $a(\mathcal{G}(\mathcal{F}))<0$ (resp. $a(\mathcal{G}(\mathcal{F}))=-2$ ). Hence the required equivalence follows, since $\mathcal{R}(\mathcal{F})=\mathcal{R}^{G}$ and $\mathcal{G}(\mathcal{F}) \cong \mathcal{G}^{G}$ by (2.2).

\section{The case where $I=\mathfrak{m}$}

We begin with a survey on canonical characters [3]. For a while let $G$ be a finite group of order $N$ and let $k$ be a field. Let $R=\oplus_{n \geq 0} R_{n}$ be a Noetherian graded ring with $R_{0}=k$. We assume the following three conditions.

(i) $R$ is a Gorenstein ring.

(ii) $G$ acts on $R$ as automorphisms of graded $k$-algebras.

(iii) $N \neq 0$ in $k$. 
Then by (iii) the graded $k$-algebra $R^{G}$ is a Cohen-Macaulay ring. Let $K_{R}$ and $K_{R} G$ denote respectively the canonical modules of $R$ and $R^{G}$ (cf. [6, Sect. 2]). Since the extension $R / R^{G}$ is module-finite, we have an isomorphism $K_{R} \cong \operatorname{Hom}_{R} G\left(R, K_{R^{G}}\right)$ of graded $R$-modules (cf. [6, (2.2.9)]). Therefore $R(a) \cong \operatorname{Hom}_{R^{c}}\left(R, K_{R^{c}}\right)$, because $K_{R}=R(a)$ by assumption (i) (here $a=a(R)$ denotes the $a$-invariant of $R$ (cf. [6, (3.1.4)])). Let $L=\operatorname{Hom}_{R} G\left(R, K_{R^{c}}\right)$ and let $\xi \in L_{-a}$ be a generator for the $R$-module $L$. Let the group $G$ act on $L$, setting $\sigma(f)=f \circ \sigma^{-1}$ for $\sigma \in G$ and $f \in L$. Choose a character $\Psi$ of $G$ over $k$ satisfying the equality

$$
\sigma(\xi)=\Psi(\sigma) \xi \text { for all } \sigma \in G .
$$

Then if one defines the action $*$ of $G$ on $K_{R}=R(a)$ so that $\sigma * x=\Psi(\sigma) \sigma(x)$ for $x \in K_{R}=R(a)$ and $\sigma \in G$, any isomorphism $K_{R} \cong \operatorname{Hom}_{R} G\left(R, K_{R^{c}}\right)$ of graded $R$-modules is compatible also with $G$-action. Hence this character $\Psi$ is independent of the choice of the elements $\xi \in L_{-a}$.

Definition 3.1. We put $\chi_{G, R}=\Psi^{-1}$ and call it the canonical character of $G$ with respect to the action on $R$.

Let us summarize below some basic results in [3] on canonical characters. The proof is standard and follows from the fact that any isomorphism $K_{R} \cong \operatorname{Hom}_{R^{c}}\left(R, K_{R^{c}}\right)$ of graded $R$-modules is compatible with $G$-action.

Proposition 3.2 ([3]). Let $a=a(R)$.

(1) $K_{R^{G}} \cong R_{\chi_{G, \Omega}}(a)$ as graded $R^{G}$-modules, where $R_{\chi_{G, R}}=\left\{f \in R \mid \sigma(f)=\chi_{G, R}(\sigma) f\right.$ for all $\sigma \in G\}$ denotes the semi-invariants in $R$ of weight $\chi_{G, R}$.

(2) $a \geq a\left(R^{G}\right)$.

(3) $\chi_{G, R}=1$ if and only if $a\left(R^{G}\right)=a$. When this is the case, $R^{G}$ is a Gorenstein ring.

(4) Let $f \in\left[R^{G}\right]_{n}(n>0)$ be $R$-regular. Then $\chi_{G, R / f R}=\chi_{G, R}$.

Proposition 3.3 ([10]). Assume $R=k\left[X_{1}, X_{2}, \ldots, X_{d}\right](d \geq 1)$ is a polynomial ring with $\operatorname{deg} X_{i}=1$ for all $1 \leq i \leq d$. Let $V=R_{1}$ and let $\rho: G \rightarrow G L(V)$ denote the representation of $G$ induced from the action on $R$. Then

$$
\chi_{G, R}(\sigma)=1 / \operatorname{det}(\rho(\sigma))
$$

for all $\sigma \in G$.

Now we assume that $A$ is a Cohen-Macaulay local ring with maximal ideal $m$ and $d=\operatorname{dim} A \geq 2$. Let the order $N$ of $G$ be invertible in $A$ and assume $G$ trivially acts on the residue class field $k=A / \mathfrak{m}$ of $A$. We put $\mathcal{R}=\mathcal{R}(\mathfrak{m})$ and $\mathcal{G}=\mathcal{G}(\mathfrak{m})$.

Firstly we shall prove the following, in which the equivalence of conditions (1) and (2) directly follows from Theorem 2.4 . 
Theorem 3.4. Let $\mathcal{G}$ be a Gorenstein ring. Consider the following three conditions:

(1) $\mathcal{R}^{G}$ is a Gorenstein ring.

(2) $\mathcal{G}^{G}$ is a Gorenstein ring of $a\left(\mathcal{G}^{G}\right)=-2$.

(3) $\chi_{G, G}=1$ and $a(\mathcal{G})=-2$.

Then one has the implications (1) $\Leftrightarrow(2) \Leftarrow(3)$. Furthermore, if $a(\mathcal{G}) \leq-2$ or if $\mathcal{G}$ is a normal ring and the extension $\mathcal{G} / \mathcal{G}^{G}$ is divisorially unramified, the above three conditions are equivalent to each other.

Proof. (3) $\Rightarrow$ (2). If $\chi_{G, \mathcal{G}}=1$, by (3.2) (3) $\mathcal{G}^{G}$ is a Gorenstein ring with $a\left(\mathcal{G}^{G}\right)=a(\mathcal{G})$. Hence $a\left(\mathcal{G}^{G}\right)=-2$.

$(2) \Rightarrow(3)$. Firstly assume that $a(\mathcal{G}) \leq-2$. Then as $a(\mathcal{G}) \geq a\left(\mathcal{G}^{\mathcal{G}}\right)$ by (3.2) (2), we get $a(\mathcal{G})=a\left(\mathcal{G}^{G}\right)=-2$ whence $\chi_{G, \mathcal{G}}=1$ by (3.2) (3). Therefore condition (3) is satisfied. Assume that $\mathcal{G}$ is a normal ring and $\mathcal{G}$ is divisorially unramified over $\mathcal{G}^{\mathcal{G}}$. Then $\chi_{G, G}=1$ because $\mathcal{G}^{G}$ is a Gorenstein ring (see the proof of [11, Theorem 2]), so that $a(\mathcal{G})=a\left(\mathcal{G}^{G}\right)=-2$ by $(3.2)(3)$.

Corollary 3.5. Suppose that $\mathcal{R}$ is a Gorenstein ring. Then the following two conditions are equivalent.

(1) $\mathcal{R}^{G}$ is a Gorenstein ring.

(2) $\chi_{G, G}=1$.

Proof. Since $A$ is Cohen-Macaulay and $\mathcal{R}$ is Gorenstein, by [9, (3.6)] $\mathcal{G}$ is a Gorenstein ring with $a(\mathcal{G})=-2$, whence the equivalence follows from (3.4).

Let $\rho: G \rightarrow G L\left(\mathrm{~m} / \mathrm{m}^{2}\right)$ be the representation of $G$ induced from the $G$-action on $A$.

Corollary 3.6. Assume that $A$ is a regular local ring. Then the following two conditions are equivalent.

(1) $\mathcal{R}^{G}$ is a Gorenstein ring.

(2) $\operatorname{dim} A=2$ and $\rho(G) \subseteq S L\left(\mathfrak{m} / \mathrm{m}^{2}\right)$.

Proof. Let $d=\operatorname{dim} A(\geq 2)$. Then since $A$ is a regular local ring, $\mathcal{G}=\mathcal{G}(\mathrm{m})$ is a polynomial ring in $d$ variables over $k=A / \mathfrak{m}$. Therefore $a(\mathcal{G})=-d \leq-2([6,(3.1 .6)])$. Consequently by (3.4) $\mathcal{R}^{G}$ is a Gorenstein ring if and only if $d=2$ and $\chi_{G, G}=1$. According to (3.3), the later condition $\chi_{G, G}=1$ is equivalent to saying that $\rho(G) \subseteq S L\left(\mathfrak{m} / \mathfrak{m}^{2}\right)$. 


\section{Examples}

In what follows, let $k$ be a field and let $R=k\left[X_{1}, X_{2}, \ldots, X_{n}\right](n \geq 1)$ be the polynomial ring in $n$ variables over $k$. We consider $R$ to be a graded ring with $R_{0}=k$ and $\operatorname{deg} X_{i}=1$ for all $1 \leq i \leq n$. Let $G$ be a finite group of order $N$ with $N \neq 0$ in $k$ and assume that $G$ acts on $R$ as automorphisms of graded $k$-algebras. Let $a(a \neq R)$ be a $G$-stable graded ideal in $R$. We put $R^{*}=R / \mathfrak{a}$ and $\mathfrak{D}=\left[R^{*}\right]_{+}$. Let $A=R_{\mathfrak{R}}^{*}$ and $\mathfrak{m}=\mathfrak{D} R_{\mathfrak{R}}^{*}$. Then the group $G$ acts on $A$ as automorphisms of rings, because the ideal $\mathfrak{M}$ is $G$-stable. We have

Lemma 4.1. The natural isomorphisms $R^{*} \cong \mathcal{G}(\mathfrak{D}) \cong \mathcal{G}(\mathfrak{m})$ are compatible with $G$ actions. Hence $R^{* G} \cong \mathcal{G}(\mathrm{m})^{G}$ as graded $k$-algebras.

Thanks to (4.1) we may apply Theorem 2.4 to the local ring $(A, \mathfrak{m})$ and get

Proposition 4.2. Assume that $R^{*}$ is a Cohen-Macaulay ring of $d=\operatorname{dim} R^{*} \geq 1$. Then

(1) $\mathcal{R}(\mathfrak{m})^{G}$ (resp. $\mathcal{R}(\mathrm{m})$ ) is a Cohen-Macaulay ring if and only if $a\left(R^{* G}\right)<0$ (resp. $\left.a\left(R^{*}\right)<0\right)$.

(2) Let $d \geq 2$. Then $\mathcal{R}(\mathrm{m})^{G}$ (resp. $\mathcal{R}(\mathfrak{m})$ ) is a Gorenstein ring if and only if $R^{* G}$ (resp. $\left.R^{*}\right)$ is a Gorenstein ring with $a\left(R^{* G}\right)=-2$ (resp. $\left.a\left(R^{*}\right)=-2\right)$.

Proof. Recall that $R^{* G}$ is a Cohen-Macaulay ring and that $A$ is a Cohen-Macaulay local ring with $d=\mathrm{ht}_{A} \mathrm{~m}$. And the assertions on $\mathcal{R}(\mathrm{m})^{G}$ follow from (2.4) and (4.1). The assertions on $\mathcal{R}(\mathrm{m})$ are due to $\left[5,(1.1)\right.$ and (1.2)], since $R^{*} \cong \mathcal{G}(\mathrm{m})$.

Let us now apply Proposition 4.2 to the following examples.

Example 4.3. Let $G=\mathcal{S}_{n}$ be the symmetric group and $1 \leq q \in \mathbb{Z}$. Assume that ch $k=0$. Let $G$ act on the polynomial ring $R=k\left[X_{1}, X_{2}, \ldots, X_{n}\right]$ so that $\sigma\left(X_{i}\right)=X_{\sigma(i)}$ for all $\sigma \in G$ and $1 \leq i \leq n$. We put $f=X_{1}^{q}+X_{2}^{q}+\ldots+X_{n}^{q}$ and $a=f R$. Then $a$ is a $G$ stable graded ideal in $R$, since $f \in R^{\mathfrak{G}}$. Let $R^{*}=R / \mathfrak{a}, \mathfrak{M}=\left[R^{*}\right]_{+}$, and $A=R_{\mathfrak{R}}^{*}$. Let $\mathfrak{m}=\mathfrak{D} R_{\mathfrak{g}}^{*}$. Then we have

Theorem 4.4. (1) Let $n \geq 2$. Then the following assertions hold true.

(a) $\mathcal{R}(\mathrm{m})$ is a Cohen-Macaulay ring if and only if $q<n$.

(b) $\mathcal{R}(\mathfrak{m})^{G}$ is a Cohen-Macaulay ring if and only if $q<n(n+1) / 2$.

(2) Let $n \geq 3$. Then the following assertions hold true.

(a) $\mathcal{R}(\mathrm{m})$ is a Gorenstein ring if and only if $q=n-2$.

(b) $\mathcal{R}(\mathrm{m})^{G}$ is a Gorenstein ring if and only if $q=[n(n+1)-4] / 2$.

Proof. The ring $R^{*}$ is Gorenstein with $\operatorname{dim} R^{*}=n-1$ and $a\left(R^{*}\right)=q-n$ (cf. [6, 
(3.1.6)]). Since $R^{G}$ is the polynomial ring in $n$ variables over $k$ and $R^{* G} \cong R^{G} / f R^{G}$, we see that $a\left(R^{G}\right)=-n(n+1) / 2$ and that $R^{* G}$ is a Gorenstein ring of $a\left(R^{* G}\right)=q-n(n+1) / 2$. Hence from (4.2) the assertions (a) and (b) in (4.4) follow.

If we take $n=q \geq 2$ in (4.4), the ring $\mathcal{R}(\mathfrak{m})$ is not Cohen-Macaulay but $\mathcal{R}(\mathfrak{m})^{G}$ is. Letting $n \geq 3$ and $q=[n(n+1)-4] / 2$, we get examples of non-Cohen-Macaulay rings $\mathcal{R}(\mathfrak{m})$ for which the invariant subrings $\mathcal{R}(\mathfrak{m})^{G}$ are Gorenstein.

Example 4.5. Let $n \geq 3$ and let $R=\mathbb{C}\left[X_{1}, X_{2}, \ldots, X_{n}\right]$ be the polynomial ring. Let $\zeta$ denote a primitive $(n-2)$-th root of unity. Let $\sigma: R \rightarrow R$ be the automorphism of $\mathbb{C}$ algebras defined by $\sigma\left(X_{i}\right)=\zeta X_{i}$ for $1 \leq i \leq n-1$ and $\sigma\left(X_{n}\right)=\zeta^{-1} X_{n}$. Let $G$ be the subgroup of Aut $\mathrm{c}_{\mathrm{c}} R$ generated by $\sigma$. We take $f=\sum_{1 \leq i \leq n} X_{i}^{n-2}$ and $a=f R$. Then $\mathcal{R}(\mathfrak{m})^{G}$ is a Gorenstein ring.

Proof. The ring $R^{*}$ is Gorenstein with $\operatorname{dim} R^{*}=n-1$ and $a\left(R^{*}\right)=-2$. Therefore by (4.2) (2) $\mathcal{R}(\mathfrak{m})$ is a Gorenstein ring too. On the other hand since $f \in R^{G}$, by (3.2) (4) we have $\chi_{G, R^{*}}=\chi_{G, R}$. Let $\tau$ be the linear transformation of $V=R_{1}$ induced from the action of $\sigma$ on $V$. Then $\operatorname{det} \tau=1$ whence $\chi_{G, R}=1$ by (3.3), so that $\chi_{G, R^{*}}=1$. Because the canonical isomorphism $R^{*} \cong \mathcal{G}(\mathfrak{m})$ is compatible with $G$-action, from (3.5) that the ring $\mathcal{R}(\mathfrak{m})^{G}$ is Gorenstein follows.

Example 4.6. Let $A$ be an arbitrary Noetherian local $\operatorname{ring}$ of $\operatorname{dim} A \geq 2$. Let $G$ be a finite group of order $N$ and assume that $G$ acts on $A$ as automorphisms of rings. Let $N$ be invertible in $A$. We choose elements $a, b$ in $A^{G}$ so that $a, b$ forms a subsystem of parameter for the local ring $A^{G}$. Let $I=(a, b) A$. Then $\mathcal{R}(I)^{G}$ is a Gorenstein ring, if so is $A^{G}$.

Proof. Let $J=(a, b) A^{G}$. Then since the extension $A / A^{G}$ is pure (cf. [8]), we have $I^{i} \cap A^{G}=J^{i}$ for all $i \in \mathbb{Z}$. Hence $\mathcal{R}(I)^{G}=\mathcal{R}(J) \cong A^{G}[X, Y] /(a X-b Y)$ (here $A^{G}[X, Y]$ is the polynomial ring in two variables over $A^{G}$ ). Thus the ring $\mathcal{R}(I)^{G}$ is Gorenstein, if so is $A^{G}$.

\section{REFERENCES}

1. I. M. Aberbach, C. Huneke and K. E. SMith, A tight closure approach to arithmetic Macaulayfication, preprint.

2. M. F. Atiyah and I. G. Macdonald, Introduction to commutative algebra (AddisonWesley, 1969).

3. S. Goтo, On Gorenstein rings (in Japanese), Sûgatiu 31 (1979), 349-364.

4. S. Goto and K. NishiDA, The Cohen-Macaulay and Gorenstein Rees algebras associated to filtrations (Mem. Amer. Math. Soc., vol. 526, Amer. Math. Soc., Providence, Rhode Island, 1994).

5. S. Goto and Y. Shimoda, On the Rees algebras of Cohen-Macaulay local rings, in Commutative Algebra, Analytic Methods (R. N. Draper, ed., Lecture Notes in Pure and Applied Mathematics, vol. 68, Marcel Dekker, Inc., New York and Basel, 1982), 201-231. 
6. S. Goto and K. Watanabe, On graded rings I, J. Math. Soc. Japan 30 (1978), 179-213.

7. M. HeRrman, S. IKEDA and U. ORBANZ, Equimultiplicity and blowing up. An algebraic study (Springer-Verlag, 1988).

8. M. Hochster and J. A. EAGoN, Cohen-Macaulay rings, invariant theory, and the generic perfection of determinational loci, Amer. J. Math. 93 (1971), 1020-1068.

9. S. IKEDA, On the Gorensteinness of Rees algebras over local rings, Nagoya Math. J. 102 (1986), 135-154.

10. K. Watanabe, Certain invariant subrings are Gorenstein, I, Osaka J. Math. 11 (1974), $1-8$.

11. K. Watanabe, Certain invariant subrings are Gorenstein, II, Osaka J. Math. 11 (1974), $379-388$.

Department of Mathematics

SCHOOL OF SCIENCE AND TECHNOLOGY

MEIJI UNIVERSTTY

214-71 JAPAN

E-mail address: s-iai@math.meiji.ac.jp 